\title{
Platelets and Neutrophils Are Critical to the Enhanced Glomerular Arachidonate Metabolism in Acute Nephrotoxic Nephritis in Rats
}

\author{
Xiaobo Wu, Jeffrey Pippin, and James B. Lefkowith \\ Departments of Medicine and Molecular Biology and Pharmacology, Washington University \\ School of Medicine, St. Louis, Missouri 63110
}

\begin{abstract}
Nephrotoxic nephritis (NTN) is characterized by a marked increase in glomerular eicosanoid synthesis, which appears to play an important role in the pathophysiology of this disease model. In this study, we investigated the biochemical and cellular basis of this metabolic change. By examining the enzymatic conversion of exogenous substrates by intact glomeruli, we found that cyclooxygenase, TX synthase, and 5-lipoxygenase activities increased 4-, 8-, and 100-fold, respectively, in acute NTN. $\mathbf{P G H}_{2}-\mathrm{PGE}_{2}$ isomerase and leukotriene $\mathrm{A}_{4}$ hydrolase activities did not change. The cellular basis of these changes was examined using dissociated glomerular cells in vitro and by depleting platelets in vivo. Dissociated glomerular cells from nephritic glomeruli (largely mesangial cells and leukocytes) exhibited an enhanced arachidonate metabolism similar to intact nephritic glomeruli. Depletion of neutrophils (PMNs) from these cell preparations by $90 \%$ commensurately decreased 5-lipoxygenase and cyclooxygenase activity but had little effect on TX synthase activity. The recovered PMN fraction, however, did exhibit TX synthase activity. Immunocytochemical analysis of dissociated cells using an antiplatelet antibody demonstrated the presence of platelets, both adherent to cells and noncell associated. Depletion of platelets in vivo using this antibody substantially attenuated the increase in glomerular eicosanoid synthesis that accompanied NTN. Platelet depletion also decreased the influx of PMNs into the glomerulus by $50 \%$. These data show that PMNs and platelets colocalize to the glomerulus in acute NTN and are coordinately essential to the increase in glomerular arachidonate metabolism. (J. Clin. Invest. 1993. 91:766-773.) Key words: neutrophils - mesangial cells • platelets $\bullet$ thromboxane $\bullet$ leukotriene
\end{abstract}

\section{Introduction}

Tissue injury is often accompanied by alterations in local eicosanoid production, which may then play a pathophysiological role in the resultant tissue damage and/or dysfunction (1). Immune-mediated glomerulonephritis in general (and nephro-

Address correspondence to Dr. James B. Lefkowith, Box 8045, Division of Rheumatology, Washington University School of Medicine, St. Louis, MO 63110

Received for publication 30 June 1992 and in revised form 2 October 1992.

J. Clin. Invest.

(C) The American Society for Clinical Investigation, Inc.

0021-9738/93/03/0766/08 \$2.00

Volume 91, March 1993, 766-773 toxic nephritis [NTN $]^{1}$ specifically) serve as examples of this phenomenon (2-12). NTN and related models of immunemediated glomerulonephritis are characterized by glomerular inflammation, marked alterations in glomerular eicosanoid production, as well as glomerular dysfunction (2-12). These alterations in glomerular eicosanoid production appear to be critically important to the pathophysiology of this disease model. Eicosanoids may mediate many of the changes in renal function (e.g., glomerular filtration and renal blood flow) that are part of the glomerular lesion in $\operatorname{NTN}(2,5,13)$. Moreover, it appears that eicosanoids may mediate or modulate the cellular component of the glomerular inflammation $(7,8,10)$. The mechanisms regulating glomerular eicosanoid synthesis in inflammation, however, remain to be fully clarified.

With respect to eicosanoids, normal glomeruli constitutively make predominantly $\mathrm{PGE}_{2}$ and smaller amounts of TX (14). The mesangial cell agonist angiotensin II selectively increases the synthesis of these cyclooxygenase metabolites, implying a mesangial cell origin for these metabolites (14). Leukotriene( $\mathrm{LT}$ ) $\mathrm{B}_{4}$ is not made constitutively by glomeruli or in response to angiotensin II but can be produced in response to calcium ionophore (15). Studies have suggested that $\mathrm{LTB}_{4}$ synthesis by normal glomeruli is largely due to the resident macrophage population (15).

With glomerular inflammation (e.g., NTN) a marked increase in products of the 5-lipoxygenase and cyclooxygenase pathways has been observed $(2,3,5,8,9,12)$. Ionophore-stimulated glomerular $\mathrm{LTB}_{4}$ rises acutely (i.e., between 1 and $6 \mathrm{~h}$ ) but returns to control levels rapidly (within $24 \mathrm{~h})(3,9)$. A more prolonged increase in constitutive or agonist-stimulated glomerular TX production is seen in NTN, which begins acutely but continues throughout the heterologous and autologous phases of the disease $(2,5,8,9)$. Interestingly, despite a severalfold increase in TX synthesis, production of the cyclooxygenase metabolite, $\mathrm{PGE}_{2}$, is only modestly affected (2, $8,9)$. Of necessity, the biochemical and cellular underpinnings of the changes seen with glomerular inflammation must be complex.

Glomerular inflammation and the accompanying changes in glomerular eicosanoid production are clearly interrelated. Several in vivo studies using a variety of manipulations have shown correlations between the inflammatory cell influx and the changes in glomerular eicosanoid production $(4,9,12)$. The consensus of these studies is that the invasion of leukocytes is necessary for the increase in glomerular $\mathrm{LTB}_{4}$ and TX production, although it is not entirely clear what the roles of the various participating leukocytes (i.e., neutrophils [PMNs] and

1. Abbreviations used in this paper: $\mathrm{CR} 1$, complement receptor 1; HRP, horseradish peroxidase; LC, leukocyte common; NTN, nephrotoxic nephritis. 
macrophages) are, nor how they may interact with resident glomerular cells or other circulating blood elements (such as platelets) to produce the changes in glomerular eicosanoid production.

The current study focuses on two issues. One is understanding the underlying alterations in glomerular arachidonate metabolism in terms of delineating the changes in the enzyme activities of the arachidonate metabolic cascade. This problem is approached by measuring the activities of the individual enzymes using the appropriate substrates (arachidonate, $\mathrm{PGH}_{2}$, and $\left.\mathrm{LTA}_{4}\right)$. Additionally, we have endeavored to correlate these biochemical changes with the changing cellular composition of the glomerulus using both glomerular cell dispersions and by depleting platelets in vivo. Using the combination of these techniques we have established that a PMN-platelet interaction is a critical component of the observed increase in both $\mathrm{LTB}_{4}$ and TX production seen in acute NTN.

\section{Methods}

Reagents and materials. Weanling Lewis rats were obtained from Charles River Breeding Laboratories, Inc. (Wilmington, MA ). Collagenase type I, trypsin type III, DNAase type III, EDTA, diaminobenzidine, $o$-phenylenediamine, and arachidonic acid were purchased from Sigma Chemical Co. (St. Louis, MO). $\mathrm{LTA}_{4}, \mathrm{LTB}_{4}$, and $\mathrm{PGH}_{2}$ were purchased from BIOMOL Research Laboratories, Inc. (Plymouth Meeting, PA ). $\left[{ }^{3} \mathrm{H}\right] \mathrm{LTB}_{4}(32 \mathrm{Ci} / \mathrm{mmol})$ was purchased from DuPont, New England Nuclear Research Products (Boston, MA). ${ }^{125} \mathrm{I}_{-} \mathrm{TXB}_{2}$ and ${ }^{125} \mathrm{I}_{-} \mathrm{PGE}_{2}$ were made as described (16). $\mathrm{TXB}_{2}$ and $\mathrm{PGE}_{2}$ antisera were kindly provided by Dr. Philip Needleman (Monsanto Co., St. Louis, MO). Antibody against $\mathrm{LTB}_{4}$ was a gift from $\mathrm{Dr}$. A. Ford-Hutchinson (Merck-Frosst, Pointe Clare-Dorval, Quebec, Canada ). Ionomycin was purchased from Calbiochem Corp. (La Jolla, CA). Mouse monoclonal anti-rat leukocyte common (LC) antigen and anti-Thy-1 antibodies were obtained from Chemicon International, Inc. (Temecula, CA). Horseradish peroxidase(HRP)-labeled rabbit anti-mouse IgG and HRP-labeled goat anti-rabbit IgG were obtained from BioRad Laboratories (Richmond, CA ) and Sigma Chemical Co., respectively. HRP-labeled rabbit antirat $\mathrm{C} 3$ was purchased from Cappel Laboratories (Malvern, PA). Hematoxylin was obtained through EM Diagnostic Systems, Inc. (Gibbstown, NJ). Organic chemicals were gas chromatography/mass spectrometry grade and were obtained from Burdick \& Jackson (Muskegon, MI).

Preparation and use of antisera. The preparation of nephrotoxic serum has been described previously ( 8 ). In short, rabbits were repeatedly immunized with glomerular basement membrane harvested from rat glomeruli ( $1 \mathrm{mg} / \mathrm{ml}$ emulsified in PBS/adjuvant 50:50). Rabbits were bled $10 \mathrm{~d}$ after immunization, and fresh serum was harvested and decomplemented. To induce nephritis, rats were given a dose of $0.5 \mathrm{ml}$ of nephrotoxic serum/ $100 \mathrm{~g}$ body wt i.v. Proteinuria was measured by the Bradford assay (Bio-Rad Laboratories). Preimmune serum had no effect on either glomerular eicosanoid production, glomerular leukocytes, or proteinuria (data not shown).

An anti-rat platelet polyclonal antibody was raised by repeatedly immunizing rabbits with $1 \times 10^{9}$ washed rat platelets (emulsified in PBS/adjuvant 50:50). Rabbits were bled $10 \mathrm{~d}$ after the final immunization, and fresh serum was harvested and decomplemented. Serum was then adsorbed against a suspension of rat erythrocytes and leukocytes. The adsorbed serum was then used in vivo to deplete platelets. Animals were injected with $2.0 \mathrm{ml}$ of antiplatelet antibody $16 \mathrm{~h}$ before induction of NTN. This dose of antibody depleted platelets by $>99 \%(<1,000 /$ $\mathrm{mm}^{3}$ ) but did not significantly affect circulating leukocyte counts $(13,100 \pm 1,000$ vs. $13,800 \pm 600$ in platelet depleted and controls, respectively). The antiplatelet antibody also did not significantly deplete circulating complement levels: $0.281 \pm 0.030$ vs. $0.277 \pm 0.060$ OD units $/ 0.5 \mu \mathrm{l}$ serum in a hemolytic assay in the two groups, respectively (CompQuick; Sigma; Chemical Co.).
We additionally assessed the effect of platelet depletion on the deposition of nephrotoxic serum and the accompanying deposition of complement in the glomerulus in NTN. Glomerular deposition of nephrotoxic serum (i.e., rabbit IgG) and rat $\mathrm{C} 3$ were measured by an antibody capture ELISA. Briefly, glomeruli were harvested from kidneys perfused free of blood with PBS, homogenized, and dilutions of the homogenate coated onto a 96-well polystyrene plate. After a 1-h incubation at room temperature, plates were washed with PBS, blocked for $1 \mathrm{~h}$ with PBS with $10 \%$ goat serum, rewashed, and then exposed to an appropriate secondary in a saturating concentration (1:1,000 dilution of the aforementioned HRP-labeled secondary antibodies). After a 1-h incubation at room temperature, plates were again washed and exposed to $o$-phenylenediamine. Plates were developed for $15 \mathrm{~min}$ and read at 490 $\mathrm{nm}$. Rabbit IgG was quantified by comparison to a standard curve. Rat $\mathrm{C} 3$ was quantified as relative OD since no standard was available. Glomerular deposition of nephrotoxic serum was: $<3,48 \pm 9$, and $62 \pm 13$ $\mu \mathrm{g} / 30 \times 10^{3}$ glomeruli in control, NTN, and NTN postplatelet-depletion animals, respectively ( $P=$ NS for NTN vs. NTN postplatelet depletion). Glomerular deposition of rat $\mathrm{C} 3$ was undetectable, $0.110 \pm 0.010$, and $0.108 \pm 020$ OD U $/ 30 \times 10^{3}$ glomeruli in control, NTN, and NTN postplatelet depletion animals, respectively $(P=$ NS for NTN vs. NTN post-platelet depletion). Thus the deposition of IgG and subsequent activation of complement were not altered by platelet depletion.

Glomerular isolation and incubations. Glomeruli were isolated from saline-perfused kidneys using a sieving protocol described previously (14). Preparations were $\sim 90 \%$ pure. Glomeruli were then permeabilized with collagenase/DNAase, rinsed with Krebs-Henseleit buffer, and incubated for two 10 -min periods at $37^{\circ} \mathrm{C}$ in Krebs-Henseleit buffer containing fatty acid-free bovine serum albumin $(1 \mathrm{mg} / \mathrm{ml})$ to preserve viability and stabilize the preparation (14). Next the glomeruli were incubated without any agonist for $10 \mathrm{~min}$ to determine basal eicosanoid production. After the determination of constitutive eicosanoid synthesis, glomeruli were exposed to one of three different substrates (arachidonate, $\mathrm{PGH}_{2}$, or $\mathrm{LTA}_{4}$ ) to assess the various enzymes of the arachidonate metabolic cascade. Alternatively, glomeruli were exposed to $1 \mu \mathrm{M}$ angiotensin II or $10 \mu \mathrm{M}$ ionomycin to determine agonist-stimulated eicosanoid production as previously detailed $(9,14)$.

To assess cyclooxygenase and 5-lipoxygenase activities, glomeruli were incubated with $10 \mu \mathrm{M}$ arachidonate. $1 \mu \mathrm{M}$ ionomycin was also added since arachidonate alone is only inefficiently metabolized via the 5-lipoxygenase pathway (15). Metabolism via 5-lipoxygenase requires coaddition of arachidonate with a low concentration of calcium ionophore (15). After $5 \mathrm{~min}$, incubations were stopped by removal of the glomeruli by centrifugation. Supernatants were then assayed for $\mathrm{PGE}_{2}$, $\mathrm{TX}$, and $\mathrm{LTB}_{4}$ by radioimmunassays as previously detailed (16).

To assess TX synthase, glomeruli were exposed to $10 \mu \mathrm{M} \mathrm{PGH}_{2}$ for $1 \mathrm{~min}$. Because of the aqueous instability of $\mathrm{PGH}_{2}$, glomeruli were added to substrate. Incubations were stopped by the addition of 1 vol ethanol followed by centrifugation. Aliquots of the supernatant were dried under $\mathrm{N}_{2}$, and TX was determined by radioimmunoassay.

LTA $_{4}$ hydrolase was measured in a similar fashion. Glomeruli were added to tubes containing $\mathrm{LTA}_{4}$ ( formed from the hydrolysis on ice of LTA $_{4}$ methyl ester) for 2 min (final concentration, $10 \mu \mathrm{M}$ ). Incubations were stopped by the addition of 1 vol ethanol. Incubations were then dried under $\mathrm{N}_{2}$, resuspended in $1 \mathrm{ml}$ of methanol/water (50:50), and fractionated by high pressure liquid chromatography to separate $\mathrm{LTB}_{4}$ from its nonenzymatic (and cross-reactive) hydrolysis products. High pressure liquid chromatographic separations were performed on a chromatograph (model 338; Beckman Instruments, Inc., San Ramon, CA) run by System Gold using a reverse-phase column ( $75 \mathrm{~mm}$ column, $3 \mu \mathrm{m}$ particle size; Beckman Instruments, Inc.) eluted at 1 $\mathrm{ml} / \mathrm{min}$ using a linear gradient of methanol into water (from 50 to $100 \%$ over $20 \mathrm{~min}$ ). Fractions comigrating with authentic $\mathrm{LTB}_{4}$ were collected, dried under $\mathrm{N}_{2}$, and assayed for $\mathrm{LTB}_{4}$ by radioimmunoassay.

Glomerular dissociation, leukocyte depletion, and cell incubations. Glomeruli were isolated as described above and dispersed into singlecell preparations using a variation of a previously published enzymatic 
dissociation protocol $(17,18)$. This type of enzymatic digestion yields a single-cell suspension of mesangial cells and leukocytes. Occasional endothelial cells are present; however, epithelial cells are largely lost during the protocol.

In the first step, glomeruli were placed in HBSS with $10 \mathrm{mM}$ Hepes containing collagenase, trypsin, and DNAase $(100,5$, and $20 \mathrm{U} / \mathrm{ml}$, respectively) and rocked at room temperature for $20 \mathrm{~min}$. Afterwards, glomeruli were pelleted by centrifugation at $500 \mathrm{rpm}$ and rinsed three times with cold HBSS without calcium or magnesium. In the second step, glomeruli were incubated in HBSS without calcium or magnesium containing $2 \mathrm{mM}$ EDTA at $37^{\circ} \mathrm{C}$ for $15 \mathrm{~min}$ with agitation. Partially intact glomeruli and single cells were subsequently removed by centrifugation. In the third step, the partially intact glomeruli and single cells were incubated in HBSS with $10 \mathrm{mM}$ Hepes containing 1 $\mathrm{mg} / \mathrm{ml}$ collagenase for $15 \mathrm{~min}$ at $37^{\circ} \mathrm{C}$, and then forced through a 22-gauge needle several times to yield a single-cell suspension. Basement membrane and debris were filtered out by passing the cells through siliconized glass wool.

Cell counts were performed after dissociation to obtain total cell yields and to assess viability (uniformly $>90 \%$ by trypan blue exclusion ). Cell dispersions were then further fractionated into adherent and nonadherent subpopulations by incubating the suspensions in a 35 mm culture dish for $1 \mathrm{~h}$ at $37^{\circ} \mathrm{C}$. Nonadherent cells were subsequently removed by agitation and gentle aspiration.

To assess cyclooxygenase and 5-lipoxygenase activity, cell suspensions were incubated in a microfuge tube (or dish for adherent cells) in a volume of $0.5 \mathrm{ml}$ to which $10 \mu \mathrm{M}$ arachidonate with $1 \mu \mathrm{M}$ ionomycin was added for $10 \mathrm{~min}$. Supernatants were assayed for $\mathrm{LTB}_{4}, \mathrm{TX}$, and $\mathrm{PGE}_{2}$ by radioimmunoassay. Cell pellets were solubilized in SDS $1 \%$ for protein assay (using the bicinchoninic acid [BCA] protein assay [Pierce Chemical Co., Rockford, IL]). Eicosanoid production was normalized to cell protein.

Leukocyte quantification and labeling for platelet deposition. Aliquots of cell suspensions ( $\sim 30,000$ cells $)$ were pelleted onto slides by cytocentrifuge (Shandon Inc., Pittsburgh, PA). The cells were fixed with methanol:acetone (50:50). Slides were labeled with an anti-LC antigen antibody (1:100 dilution), washed, and then labeled with a rabbit anti-mouse antibody conjugated to HRP (1:100 dilution). Slides were washed and bound antibody was visualized with diaminobenzidine. Hematoxylin was used as a counterstain. The positively labeled cells were quantified as a percentage of the total by light microscopy and categorized by nuclear morphology as to lineage. Leukocytes counts per glomerulus were then calculated by multiplying leukocyte percentages (or subset fractions) by the total glomerular cell number. This technique correlates well with other methods of leukocyte quantification, such as in situ staining (10).

The above protocol was used to assess the presence of platelets in glomerular cell dissociates with the only change being the use of the aforementioned antiplatelet polyclonal antibody as the detecting reagent. This polyclonal antibody was purified using protein A-Sepharose (Pharmacia LKB Biotechnology Inc., Uppsala, Sweden) and directly labeled with HRP using a kit from Zymed Laboratories Inc. (South San Francisco, CA). Platelets were identified by positive staining as well as appropriate morphology.

Statistical analysis. Statistical analysis was performed with the aid of STATA (Computing Resource Center, Los Angeles, CA). Values are expressed as mean \pm SE. Each replicate represents the data from both kidneys from one animal. Two-way comparisons were made with Student's $t$ test. When comparisons were made between multiple groups, analysis of variance was performed along with Bonferroni's correction for multiple comparisons. Significance at the $P<0.05$ level is indicated in the text or figure legends.

\section{Results}

Biochemical basis of the alterations in glomerular arachidonate metabolism in NTN. To delineate changes in arachidonate metabolism in acute NTN (i.e., $3 \mathrm{~h}$ after disease induction), we initially assessed flux through the cyclooxygenase and 5-lipoxygenase pathways by providing exogenous arachidonate. As shown in Fig. $1 \mathrm{~A}$, production of $\mathrm{PGE}_{2}$ from exogenous substrate increased twofold in NTN relative to control. TX synthesis was increased $\sim 10$-fold in acute NTN (Fig. $1 A$ ). Total cyclooxygenase activity (estimated by the conversion of arachidonate to both $\mathrm{PGE}_{2}$ and TX) increased three- to fourfold (Fig. $1 B)$. With exogenous arachidonate, the 5-lipoxygenase pathway was a minor one in normal glomeruli. In acute NTN, however, 5-lipoxygenase activity increased 100 -fold and became the dominant metabolic pathway (Fig. $1 A$ ).

As noted in Fig. $1 B$, not only did total cyclooxygenase activity increase with NTN (in terms of $\mathrm{PGE}_{2}$ and TX synthesis), but the ratio between TX and $\mathrm{PGE}_{2}$ increased approximately sevenfold. Since TX and $\mathrm{PGE}_{2}$ are products of the common intermediate $\mathrm{PGH}_{2}$, this latter observation indicates a change in the enzymes downstream from the cyclooxygenase: TX synthase and/or $\mathrm{PGH}_{2}-\mathrm{PGE}_{2}$ isomerase. We thus subsequently assessed the effects of glomerular inflammation on TX synthase by examining the conversion of $\mathrm{PGH}_{2}$ by glomeruli.

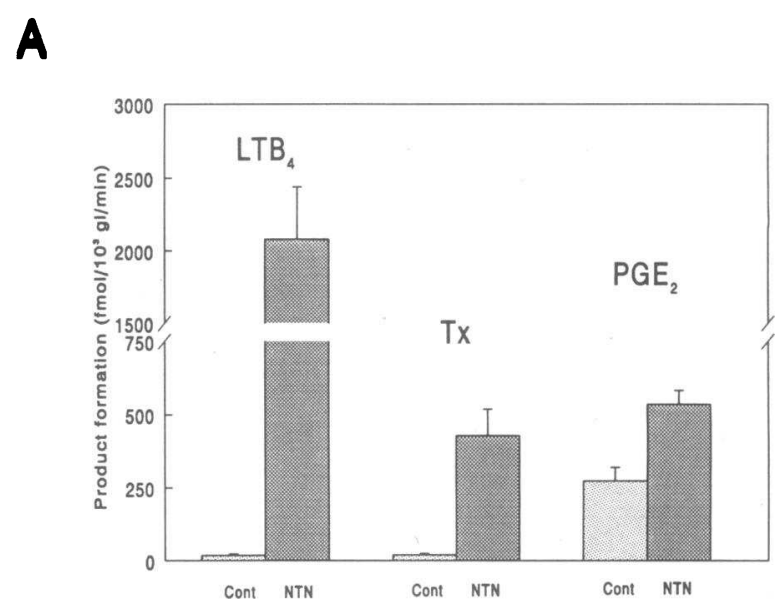

B

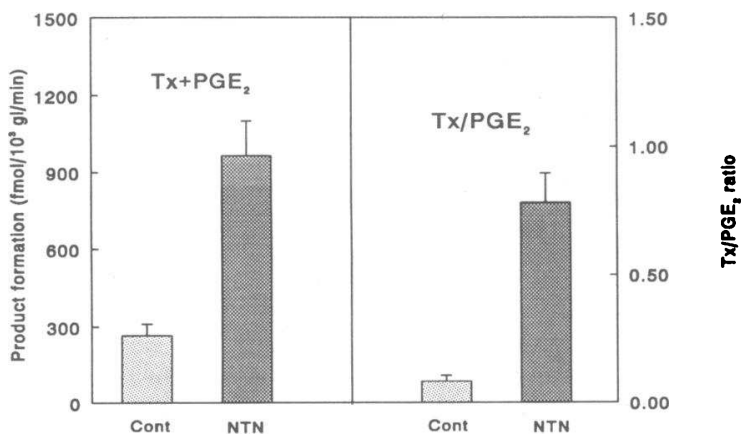

Figure 1. Conversion of exogenous arachidonate via cyclooxygenase and 5-lipoxygenase pathways by glomeruli. Glomeruli from control and nephritic rats were isolated and the conversion of exogenous arachidonate via cyclooxygenase and 5-lipoxygenase pathways was determined as detailed in Methods. Eicosanoid measurements were made by specific radioimmunoassay and normalized for the number of glomeruli and incubation time. $n=4$ and 3 for control and NTN, respectively. Control was significantly different than NTN for all comparisons $(P<0.05)$. $(A)$ Individual eicosanoid measurements; $(B)$ cyclooxygenase activity (as the sum of TX and $\mathrm{PGE}_{2}$ ) and TX synthase activity (as the ratio of TX to $\mathrm{PGE}_{2}$ ). 


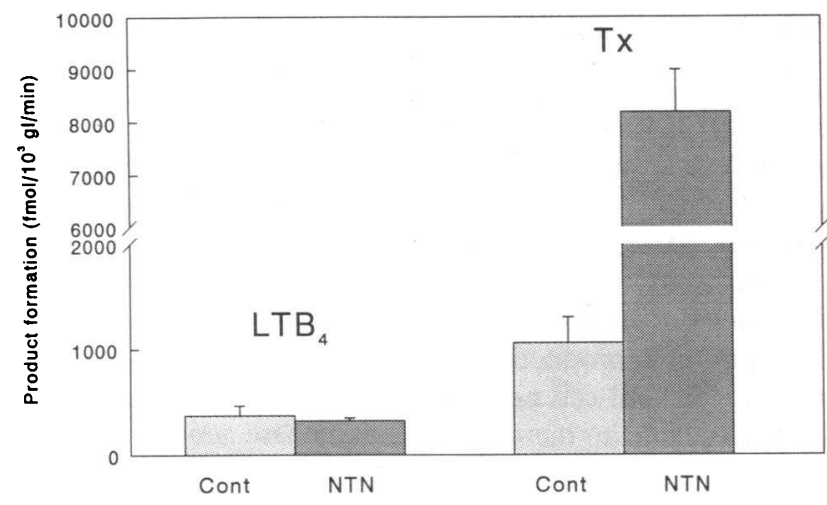

Figure 2. Conversion of $\mathrm{LTA}_{4}$ and $\mathrm{PGH}_{2}$ by glomeruli. Glomeruli from control and nephritic rats were isolated and the conversion of exogenous $\mathrm{LTA}_{4}$ and $\mathrm{PGH}_{2}$ to $\mathrm{LTB}_{4}$ and TX, respectively, was determined as detailed in Methods. $n=3$ for all groups. Control was significantly different than NTN only for TX $(P<0.05)$.

As shown in Fig. 2, TX synthase activity increased approximately eightfold in acute NTN. We used the experimentally determined rates for TX synthase and the ratios of TX/ $\mathrm{PGE}_{2}$ derived from the basal incubation period before $\mathrm{PGH}_{2}$ exposure to then calculate the rate of the $\mathrm{PGH}_{2}-\mathrm{PGE}_{2}$ isomerase ( since $\mathrm{PGE}_{2}$ and TX are produced frcm the common intermediate $\mathrm{PGH}_{2}$ ). The calculated rates for the isomerase were $15,356 \pm 3,567$ and $10,644 \pm 1,045 \mathrm{fmol} / 10^{3}$ glomeruli per min in control and nephritic glomeruli, respectively $(n=3, \mathrm{p}$ $=\mathrm{NS})$. Activity of the $\mathrm{PGH}_{2}-\mathrm{PGE}_{2}$ isomerase was thus not affected by glomerular inflammation. Moreover, the ratio of $\mathrm{TX} / \mathrm{PGE}_{2}$ reflected changes in TX synthase determined directly.

The increase in the activity of the 5-lipoxygenase pathway was further investigated by examining the downstream enzyme, $\mathrm{LTA}_{4}$ hydrolase. As shown in Fig. 2, glomerular $\mathrm{LTA}_{4}$ hydrolase was unaffected by the induction of glomerular inflammation. Therefore, although in normal glomeruli the 5-lipoxygenase was the apparent rate-limiting step for its respective pathway, the glomerular $\mathrm{LTA}_{4}$ hydrolase became the apparent rate-limiting step of the 5-lipoxygenase pathway in the context of acute NTN.

In sum, the glomerular inflammation of acute NTN was characterized by a 3- to 4-fold increase in cyclooxygenase, an 8-fold increase in TX synthase, and a 100-fold increase in 5-lipoxygenase with no apparent change in the $\mathrm{PGH}-\mathrm{PGE}_{2}$ isomerase or $\mathrm{LTA}_{4}$ hydrolase.

Cellular correlates of the changes in glomerular arachidonate metabolism in acute NTN. We subsequently investigated the cellular basis of the above changes in glomerular arachidonate metabolism using glomerular cell dispersions. Dissociation of normal glomeruli yielded $\sim 120$ cells per glomerulus containing 16\% leukocytes (LC antigen-positive cells) (Fig. $3 \mathrm{~A}$ ). The remainder of the cells were largely mesangial cells (80$90 \%$ Thy- 1 positive by immunocytochemistry). The leukocytes present in normal glomeruli were almost exclusively macrophages (Fig. $3 B$ ). In acute NTN, glomeruli were clearly hypercellular and yielded 192 cells per glomerulus upon dispersion (Fig. $3 \mathrm{~A}$ ). This increase was solely due to an increase in leukocytes (Fig. $3 A$ ). Mesangial cell number remained constant (Fig. $3 A$ ). The leukocytes present in acutely inflamed glomeruli were largely PMNs (Fig. $3 \mathrm{~B}$ ). Macrophages comprised only $20 \%$ of the leukocytes present in inflamed glomeru- lar dispersions and were present in numbers comparable to those in normal glomeruli (Fig. $3 B$ ).

We subjected these cell dispersions to short-term culture to deplete leukocytes by adherence. As shown in Fig. $3 B$, adherence of cells from the normal glomerulus did not significantly remove the resident macrophages. In glomerular cell preparations from inflamed glomeruli, however, adherence depleted leukocytes by $70 \%$ (Fig. $3 \mathrm{~B}$ ). This result was due to the depletion of PMNs ( $>90 \%$ decrease; Fig. $3 \mathrm{~B}$ ). As with normal glomeruli, adherence was ineffective in removing the macrophage subpopulation (Fig. $3 B$ ). Both nonadherent and adherent cells were recovered for study from dissociations of inflamed glomeruli. The nonadherent subfraction consisted largely of mesangial cells ( Thy 1-positive cells) along with macrophages ( $\mathrm{LC}$ antigen-positive cells with appropriate nuclear

A

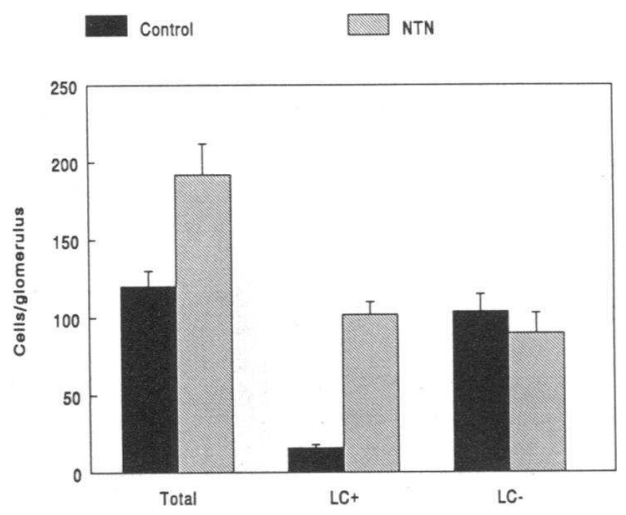

B

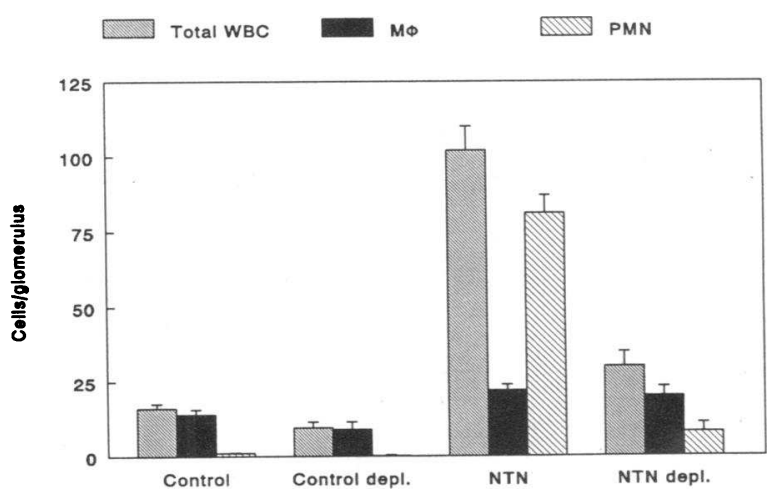

Figure 3. Composition of glomerular cell dispersions. Glomeruli from control and nephritic animals were dissociated into single cells as detailed in Methods. Leukocytes were then quantified in these dispersions by immunocytochemistry (using an anti-LC antigen antibody) and typed by nuclear morphology. Dispersions were then depleted of leukocytes by adherence and reanalyzed by immunocytochemistry. $(A)$ Characterization of the dispersions from control and nephritic glomeruli in terms of total cell yield and numbers of LC antigen-positive and negative subsets. $n=6$ for all groups. NTN was significantly different than control for total cells and the LC antigen-positive subset $(P<0.05)$. $(B)$ Leukocyte quantitation and subset analysis in control, nephritic, leukocyte-depleted control (control depl.), and leukocyte-depleted nephritic ( NTN-depl.) preparations. $n=6$ for all groups. For total white blood cells and PMNs, NTN was significantly different from both control and NTN depleted $(P<0.05)$. 
morphology). The adherent fraction was comprised solely of PMNs (> 90\% LC antigen-positive cells with appropriate nuclear morphology).

The arachidonate metabolism of cell dispersions was examined by providing exogenous substrate as with whole glomeruli above. As shown in Fig. $4 \mathrm{~A}$, cell dispersions from normal glomeruli made small amounts of $\mathrm{LTB}_{4}$ (which were just detectable ) and small amounts of TX. $\mathrm{PGE}_{2}$ was the dominant product from these cell dispersions (Fig. $4 A$ ). Arachidonate metabolism by the nonadherent cells was comparable to the unfractionated population (Fig. $4 \mathrm{~A}$ ).

Cell preparations from nephritic glomeruli exhibited a marked enhancement in eicosanoid synthesis relative to preparations from normal glomeruli: $\mathrm{LTB}_{4}$ production was increased 30-fold; TX production was increased 25-fold; and $\mathrm{PGE}_{2}$ production was increased 4-fold (Fig. $4 \mathrm{~A}$ ). Total cyclooxygenase activity (the sum of the conversion of arachidonate to TX and $\mathrm{PGE}_{2}$ ) also rose four-fold (Fig. $4 \mathrm{~B}$ ). As noted
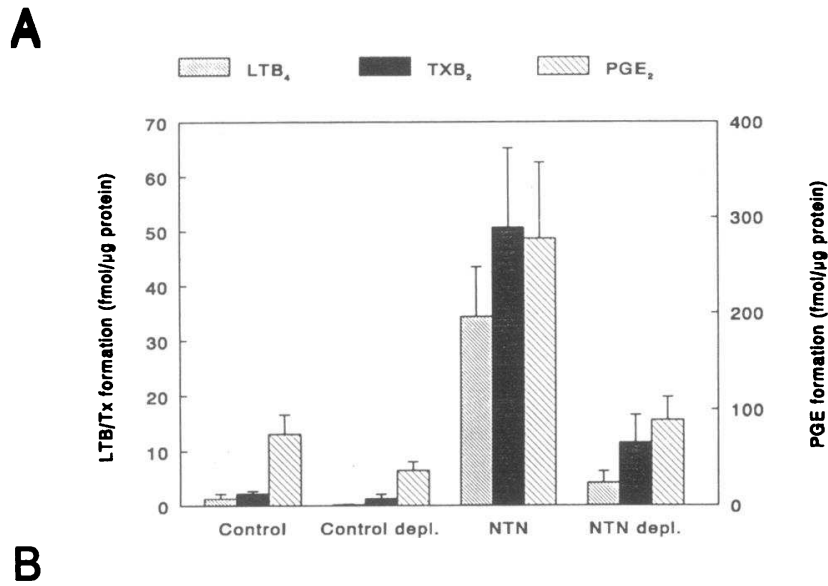

B

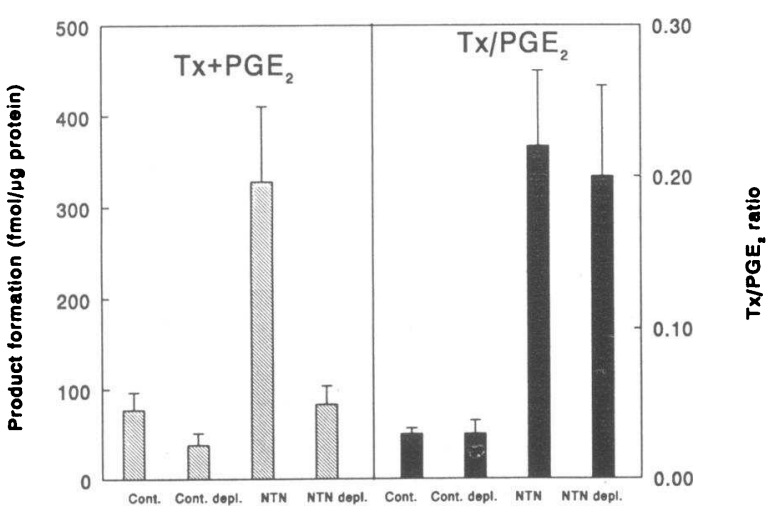

Figure 4. Conversion of exogenous arachidonate via cyclooxygenase and 5-lipoxygenase pathways by dissociated glomerular cells. The conversion of exogenous arachidonate via the cyclooxygenase and 5-lipoxygenase pathways by dissociated glomerular cells from control and nephritic glomeruli was determined as detailed in Methods. Both unfractionated dispersions (control and NTN) and dispersions depleted of leukocytes by adherence (control and NTN-depleted [depl.]) were examined. Eicosanoids were measured by specific radioimmunoassay and normalized for cell protein. $n=5$ for all groups. NTN was significantly different from control and NTN-depleted for all eicosanoid measurements except the TX/PGE 2 ratio $(P<0.05)$. $(A)$ Individual eicosanoid measurements; $(B)$ cyclooxygenase activity (as the sum of TX and $\mathrm{PGE}_{2}$ ) and TX synthase activity (as the ratio of TX to $\mathrm{PGE}_{2}$ ). above, the ratio of TX to $\mathrm{PGE}_{2}$ reflects changes in $\mathrm{TX}$ synthase activity in acute NTN. From Fig. $4 A$, it was calculated that the relative TX synthase activity rose 10 -fold in cell dispersions from nephritic glomeruli (Fig. $4 B$ ). Thus, arachidonate metabolism by the glomerular cell dispersions largely paralleled the results derived from whole glomerular incubations detailed above (Fig. 1).

Removal of PMNs from cell preparations from nephritic glomeruli by adherence ( $90 \%$ depletion) led to a comparable decrease in $\mathrm{LTB}_{4}$ production (90\% decrease) (Fig. $4 \mathrm{~A}$ ). Moreover, the adherent cell population (which consisted solely of PMNs) exhibited an increased 5-lipoxygenase activity relative to the unfractionated cells $\left(\mathrm{LTB}_{4} 59 \pm 13 \mathrm{fmol} / \mu \mathrm{g}\right.$ cell protein, $n=4, \mathrm{p}=0.06$ vs. unfractionated cells from nephritic glomeruli). Thus, the presence of PMNs and 5-lipoxygenase activity appeared covariant in these cell preparations.

PMN depletion was also effective in decreasing the conversion of arachidonate by cyclooxygenase in these cell dispersions (Fig. 4, $A$ and $B$ ). TX and $\mathrm{PGE}_{2}$ production (and their sum) were decreased to levels only slightly above control. Substantial cyclooxygenase activity was also recovered with the adherent PMNs fraction ( $321 \pm 97 \mathrm{fmol} / \mu \mathrm{g}$ cell protein $)$.

With respect to TX synthase activity, the ratio of TX to $\mathrm{PGE}_{2}$ was not significantly affected by PMN depletion of cell dispersions from nephritic glomeruli. This result, however, implies that PMNs contribute to the increase in TX synthase activity rather than not. If PMNs made no contribution, then the TX synthase activity should rise in the nonadherent cell fraction and be absent from the adherent fraction (i.e., there would be an enrichment of TX synthase specific activity by removal of noncontributing cells). Such enrichment of 5-lipoxygenase activity in the adherent PMNs was observed above. The contribution of PMNs to TX synthase in cell dispersion from nephritic glomeruli was corroborated by the observation that the adherent cell fraction (i.e., PMNs) did make substantial TX ( $44 \pm 8 \mathrm{fmol} / \mu \mathrm{g}$ cell protein) and exhibited a $\mathrm{TX} / \mathrm{PGE}_{2}$ ratio comparable to the unfractionated population $(0.25 \pm 0.04)$. This contribution must of necessity be indirect, however, in that PMNs lack the capacity to make TX (19).

Role of platelets in the enhanced glomerular eicosanoid synthesis in NTN. Because platelets produce prodigious amounts of TX, we conjectured that platelets might contribute to the alterations in glomerular eicosanoid during NTN, particularly with respect to TX synthase. In consequence, we next addressed the role of platelets in the enhanced glomerular eicosanoid synthesis in acute NTN using an antiplatelet antibody as an immunocytochemical probe in the glomerular cell dissociations and to deplete platelets in vivo before disease induction.

Immunocytochemical analysis of the above glomerular dissociates from nephritic rats using the HRP-labeled antiplatelet antibody showed the presence of $15 \pm 1$ platelets per 100 cells $(n$ $=3$ ). Platelets were found both singly and in aggregates. The majority of platelets were found in association with PMNs $(43 \pm 5 \%)$. The rest of the platelets were either unassociated with cells $(39 \pm 6 \%)$ or associated with non-PMNs (i.e., either macrophages or mesangial cells, $18 \pm 3 \%$ ). Glomerular cell dissociates from animals who had been platelet-depleted in vivo before the induction of NTN showed $<1$ platelet per 100 cells. These data suggested that platelet deposition within glomeruli might contribute to the increased TX synthase observed in NTN.

We addressed this possibility by subsequently examining the effect of the anti-platelet antibody on the enhanced glomer- 
ular eicosanoid production that accompanies NTN. In accordance with previous observations $(8,9)$, constitutive glomerular TX production increased substantially with NTN (Fig. 5 $A$ ). Platelet depletion before induction of NTN decreased the production of TX by glomeruli by $\sim 75 \%$ (Fig. $5 A$ ). Glomerular $\mathrm{PGE}_{2}$ production was not substantially altered by the induction of NTN or by platelet depletion (Fig. $5 A$ ). Using the sum of TX and $\mathrm{PGE}_{2}$ as an index of total cyclooxygenase activity, however, it was apparent that platelet depletion diminished total cyclooxygenase activity (Fig. $5 \mathrm{~B}$ ). Moreover, using the ratio of $T X$ to $\mathrm{PGE}_{2}$ as an index of $\mathrm{TX}$ synthase activity, we additionally found that platelet depletion markedly diminished the increase in TX synthase that occurred in acute NTN (Fig. 5 $B)$. Identical results were obtained if angiotensin II-stimulated TX and $\mathrm{PGE}_{2}$ production were used in lieu of constitutive production (data not shown). Thus, platelets appeared to be

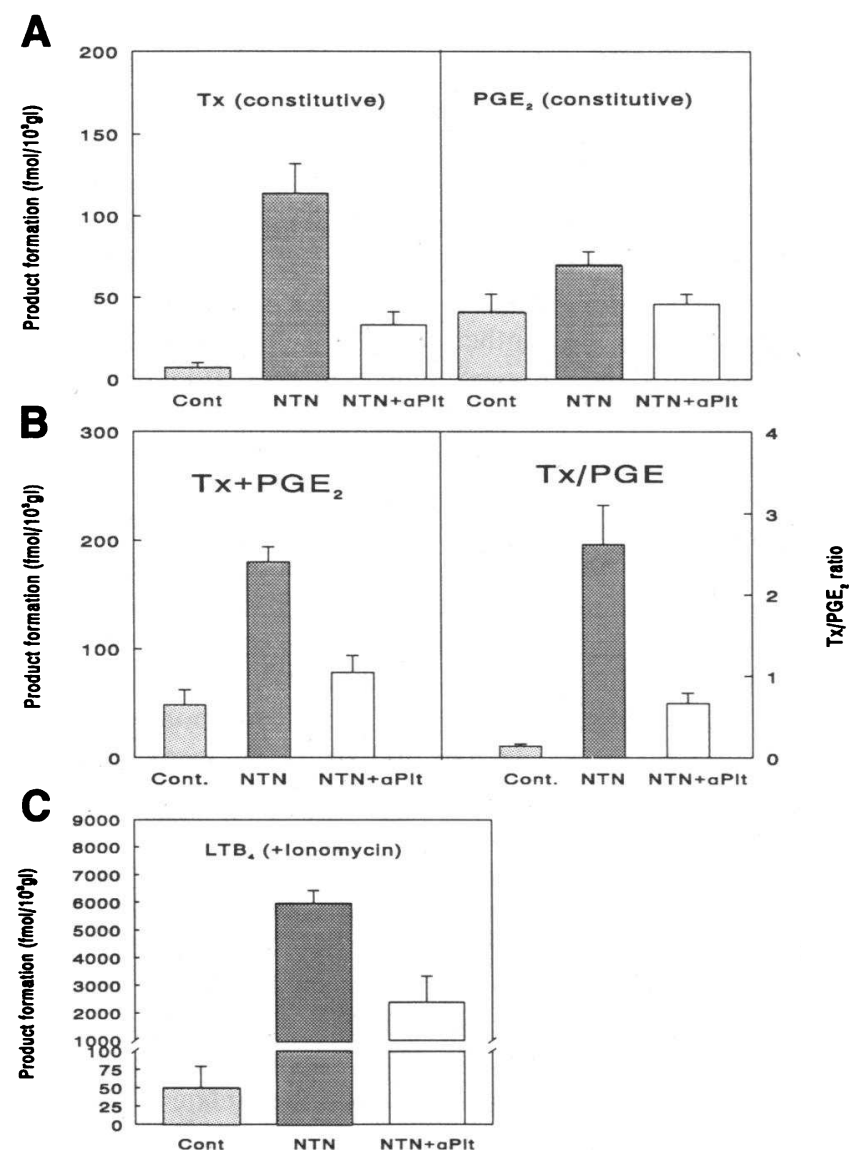

Figure 5. Effect of platelet depletion on glomerular eicosanoid synthesis in NTN. Constitutive and agonist-induced eicosanoid production were determined in intact glomeruli from control animals and nephritic animals as detailed in Methods. A subgroup of nephritic animals were platelet depleted in vivo using a polyclonal antiplatelet antibody before the induction of nephritis (NTN $+\alpha \mathrm{PLT}) . n=4$, 10 , and 4 for the three groups (control, NTN and NTN $+\alpha$ PLT). $(A)$ Constitutive glomerular TX and $\mathrm{PGE}_{2}$ production. NTN was signifcantly different from control and NTN $+\alpha$ PLT for TX only $(P$ $<0.05)$. ( $B)$ Glomerular cyclooxygenase and TX synthase activities as estimated by the sum of TX and $\mathrm{PGE}_{2}$ production and their ratio, respectively. NTN was significantly different from control and NTN $+\alpha$ PLT for both $(P<0.05)$. ( $C)$ Ionomycin-stimulated glomerular $\mathrm{LTB}_{4}$ production. NTN was significantly different from control and NTN $+\alpha$ PLT $(P<0.05)$. essential to both the increase in glomerular cyclooxygenase and TX synthase in NTN.

With respect to the 5-lipoxygenase pathway, platelet depletion decreased ionomycin-stimulated $\mathrm{LTB}_{4}$ production by $\sim 60 \%$ (Fig. $5 \mathrm{C}$ ). Constitutive $\mathrm{LTB}_{4}$ formation was also detectible in NTN but not in either controls or platelet-depleted nephritic animals (data not shown). Since platelets lack a 5-lipoxygenase (1), their contribution to glomerular 5-lipoxygenase activity must be indirect. In light of the above studies suggesting that the PMN is the source of glomerular $\mathrm{LTB}_{4}$, we conjectured that platelet depletion might be affecting the migration of PMNs. We thus assessed the effect of platelet depletion on the glomerular inflammatory cell infiltrate. As shown in Fig. 6, acute NTN was characterized by a substantial inflammatory cell influx that was largely composed of PMNs. With platelet depletion there was a modest decrease in the inflammatory cell influx; however, there was a marked change in its composition (Fig. 6). PMN influx was decreased by $50 \%$, whereas macrophage influx increased.

Notably, platelet depletion attenuated the inflammatory injury in acute NTN in terms of proteinuria. Proteinuria over the $24 \mathrm{~h}$ subsequent to disease induction was $322 \pm 54 \mathrm{mg}$ in acute NTN vs. $80 \pm 32 \mathrm{mg}$ in NTN postplatelet depletion $(n=11$ and 4 , respectively, $\mathrm{p}<0.05,5 \pm 1 \mathrm{mg}$ in controls).

\section{Discussion}

NTN and related models of glomerulonephritis are characterized by a marked enhancement in glomerular eicosanoid synthesis (2-12). Glomerular eicosanoids may then act as paracrine regulators of many of the pathophysiological alterations that accompany the disease process $(2,5,7,8,10,13)$. In this study we have addressed the biochemical and cellular mechanisms underlying the altered glomerular eicosanoid production in acute NTN.

Using both exogenous arachidonate and $\mathrm{LTA}_{4}$, we observed that the major change in the glomerular 5-lipoxygenase pathway in the context of inflammation was an increase in 5-lipoxygenase activity. LTA $_{4}$ hydrolase activity was unchanged by NTN. This change in 5-lipoxygenase activity appeared to result from the influx of PMNs into the glomerulus. This assertion is most directly supported by the cell-dissociation experiments in which the depletion of PMNs from cell preparations from inflamed glomeruli commensurately diminished $\mathrm{LTB}_{4}$ production and in which the adherent cell fraction (composed of PMNs) made substantial amounts of $\mathrm{LTB}_{4}$. These data validate in vivo data that show a correlation between glomerular PMN numbers and $\mathrm{LTB}_{4}$ synthesis in NTN $(6,9)$. Although the glomerular dissociates used in this study did not contain glomerular endothelial and epithelial cells, it is unlikely that these cells contribute to alterations in glomerular 5-lipoxygenase, since these cells do not express the 5-lipoxygenase either constitutively or in response to inflammatory mediators (20). From the available data, we would surmise that the increase in glomerular 5-lipoxygenase activity in NTN results directly from the importation of this enzyme into the glomerulus by PMNs.

The present study also shows that, at the level of intact glomeruli, the 5-lipoxygenase is the rate-limiting step in LT synthesis in normal glomeruli. This contrasts with data on single cells, in which the $\mathrm{LTA}_{4}$ hydrolase appears rate limiting (1). This difference between single cells and intact glomeruli is prob- 


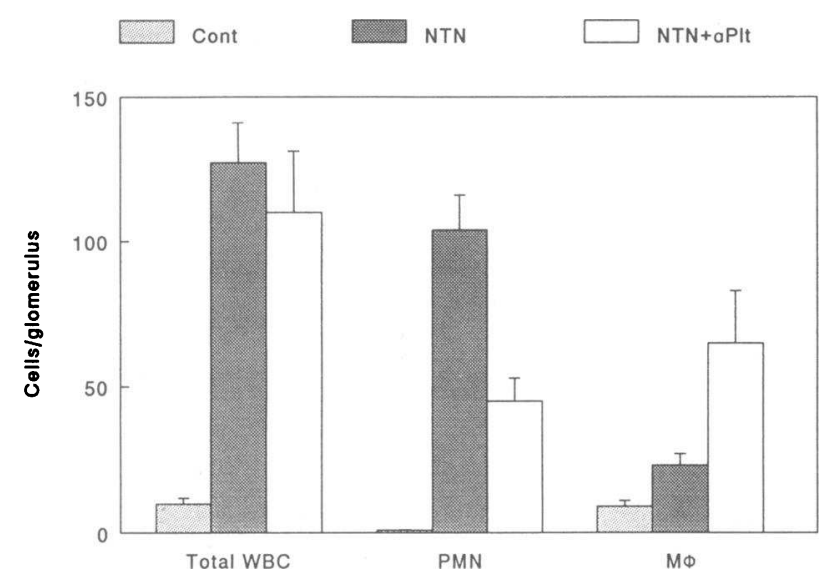

Figure 6. Effect of platelet depletion on glomerular leukocyte influx in NTN. Glomerular leukocytes and subsets (total white blood cells, $\mathrm{PMN}$, and macrophages $\mathrm{M} \phi$ ) were determined in control and nephritic animals by immunocytochemistry as detailed in Methods. A subgroup of nephritic animals were platelet depleted in vivo using a polyclonal antiplatelet antibody (NTN $+\alpha \mathrm{PLT}) \cdot n=4,10$, and 4 for the three groups (control, NTN, and NTN postplatelet depletion). NTN was significantly greater than control for both total WBC and PMN $(P<0.05)$. NTN was significantly greater than NTN $+\alpha$ PLT for PMNs $(P<0.05)$.

ably due to the presence of $\mathrm{LTA}_{4}$ hydrolase activity in glomerular cells that lack a 5-lipoxygenase (i.e., mesangial or endothelial cells) (21), thus providing whole glomeruli with an excess hydrolase capacity. Although the 5-lipoxygenase was apparently the rate-limiting step in $\mathrm{LTB}_{4}$ synthesis in normal glomeruli, $\mathrm{LTA}_{4}$ hydrolase activity was not increased in NTN, and this enzyme became the apparent rate-limiting step in the context of inflammation. These data imply that inhibiting the $\mathrm{LTA}_{4}$ hydrolase, as opposed to the 5-lipoxygenase, may have an advantage as a pharmacological strategy with respect to antiinflammatory therapy.

The data additionally suggest that platelets participate in the increase in glomerular 5-lipoxygenase activity in NTN in that platelet depletion attenuated this increase. A similar observation has been made in anti-Thy 1 -induced nephritis (4). The effect of platelet depletion appeared to be an indirect one in the current study, mediated through a decrease in PMN influx. Platelet depletion diminished both glomerular $\mathrm{LTB}_{4}$ synthesis and PMN influx equivalently. The implication of these observations is that PMN influx into glomeruli in acute NTN is in part platelet dependent. A platelet dependence for PMN influx has been noted previously in some, though not all, models of immune complex nephritis $(22,23)$.

The contribution of macrophages to glomerular $\mathrm{LTB}_{4} \mathrm{syn}-$ thesis in acute NTN appears modest. This study, and previous ones $(9,15)$, suggest that the synthetic capacity of the resident macrophage is much more limited than that of the invading PMNs. Additionally, the data derived from platelet depletion suggest that macrophages that are elicited into the glomerulus in glomerular inflammation have little capacity to produce $\mathrm{LTB}_{4}$. The limited capacity of elicited macrophages to produce LTB $_{4}$ is supported by other investigations on the heterologous and autologous phases of NTN $(9,24)$.

With respect to the cyclooxygenase pathway in acute NTN, we found that both glomerular cyclooxygenase and TX synthase activities were increased. The increase in cyclooxygenase appeared to depend in part on both PMNs and platelets. PMN depletion of dissociated glomerular cells from nephritic glomeruli and in vivo platelet depletion in acute NTN both attenuated the increase in glomerular cyclooxygenase activity. It is impossible to discern the relative contributions of each cell type from the current data since platelet depletion also apparently affected PMN migration and thus may have indirectly affected cyclooxygenase activity. With respect to TX synthase, the data on platelet depletion suggest that platelets make a major contribution to the increase seen in acute NTN. Although the cell dissociation experiments suggest that PMNs contribute to the increase in TX synthase, PMNs lack the capacity to make TX (19). The TX synthase activity in the PMN fraction of dissociated cells from nephritic glomeruli likely reflects platelet adherence to PMNs as suggested by the immunocytochemical studies using the antiplatelet antibody.

The present study, however, does not entirely rule out a contribution of glomerular mesangial, epithelial, or endothelial cells to the acute alterations in the cyclooxygenase pathway in NTN. As noted above and previously (8), angiotensin-induced eicosanoid production is markedly elevated in NTN. Since angiotensin II is a specific mesangial cell agonist (25), it is clear that mesangial cells can contribute to the enhanced glomerular eicosanoid production in NTN, possibly via release and transcellular metabolism of arachidonate. Moreover, it has been observed that mesangial cell cyclooxygenase expression can be upregulated by cytokines (26). Additionally, complement activation may induce the synthesis of TX by glomerular epithelial cells (27). Thus, the observed changes in glomerular cyclooxygenase and TX synthase activities in acute NTN may involve the resident glomerular cells in addition to the invading PMNs and platelets.

The data, however, do suggest that platelets and PMNs are important for the full expression of the changes in arachidonate metabolism in acute NTN. Moreover, the data suggest that platelets play a role in PMN migration into glomeruli and that platelets may be imported into the glomerulus via their adherence to PMNs. There are several possible (and nonexclusive) mechanisms by which platelets and PMNs may colocalize to the glomerulus. This interaction may be mediated via complement components. This assertion is supported by studies that show that complement depletion prevents the influx of PMNs into the glomerulus in acute NTN (9) and the glomerular deposition of platelets in anti-Thy 1 nephritis (28). A role for complement receptor 1 (CR1) is also suggested by the observations that both platelets and neutrophils have CR1 (29) and that soluble CR1 exerts a protective effect in immune complex injury (30). Alternatively, the interaction may relate to the propensity of platelets to spontaneously adhere to PMNs probably via fibrin/fibrinogen (19). Fibrin/fibrinogen deposition appears to be an early event in immune complex tissue injury (31). Moreover, both platelets and PMNs have receptors that may recognize fibrinogen (integrins GPIIb/IIIa $\left[\alpha_{\mathrm{IIb}} \beta_{3}\right]$ and Mac-1 $\left[\alpha_{\mathrm{M}} \beta_{2}\right]$, respectively) (32). Additionally, platelets may adhere directly to PMNs via the P-selectin, GMP140/PADGEM/CD62 (32). Regardless of the mechanism, it is clear from this study and studies on leukocyte depletion (9) that both platelets and PMNs contribute to the acute inflammatory injury in terms of proteinuria.

In conclusion, acute NTN is accompanied by an increase in glomerular 5-lipoxygenase, cyclooxygenase, and TX synthase activities. A cooperative interaction between PMNs and platelets appears to be critical to this process. The increase in glo- 
merular 5-lipoxygenase (and to a certain extent, cyclooxygenase) activity appears to occur as a direct result of the influx of PMNs into the glomerulus, this influx being in part dependent on platelets. Platelets are important for the increase in glomerular TX synthase activity in NTN and may be imported into the glomerulus via their adherence to PMNs. Both cell types contribute to the acute glomerular damage that occurs. These data emphasize the important role that PMNs and platelets play in acute immune-mediated glomerular injury and suggest an interesting parallelism with the process of hemostasis.

\section{Acknowledgments}

This work was supported by National Institutes of Health grant DK37879 (J. B. Lefkowith). Dr. Lefkowith is a Burroughs-Wellcome Scholar in Clinical Pharmacology.

\section{References}

1. Needleman, P., J. Turk, B. A. Jakschik, A. R. Morrison, and J. B. Lef kowith. 1986. Arachidonic acid metabolism. Annu. Rev. Biochem. 55:69-102.

2. Lianos, E. A., G. A. Andres, and M. J. Dunn. 1983. Glomerular prostaglandin and thromboxane synthesis in rat nephrotoxic serum nephritis. J. Clin. Invest. 72:1439-1448.

3. Lianos, E. A. 1988. Synthesis of hydroxyeicosatetraenoic acids and leukotrienes in rat nephrotoxic serum glomerulonephritis. J. Clin. Invest. 82:427-435.

4. Lianos, E. A., B. A. Bresnahan, and C. Pan. 1991. Mesangial cell immune injury: synthesis, origin, and role of eicosanoids. J. Clin. Invest. 88:623-631.

5. Takahashi, K., G. F. Schreiner, K. Yamashita, B. W. Christman, I. Blair, and K. Badr. 1990. Predominant functional roles for thromboxane $A_{2}$ and prostaglandin $\mathrm{E}_{2}$ during late nephrotoxic serum glomerulonephritis in the rat. J. Clin. Invest. 85:1974-1982.

6. Yared, A., C. Albrightson-Winslow, D. Griswold, K. Takahashi, A. Fago, and K. F. Badr. 1991. Functional significance of leukotriene $B_{4}$ in normal and glomerulonephritic kidneys. J. Am. Soc. Nephrol. 2:45-56.

7. Takahashi, K., T. Kato, G. F. Schreiner, J. Ebert, and K. F. Badr. 1992. Essential fatty acid deficiency normalizes function and histology in rat nephrotoxic nephritis. Kidney Int. 41:1245-1253.

8. Schreiner, G. F., B. Rovin, and J. B. Lef kowith. 1989. The anti-inflammatory effects of essential fatty acid deficiency in experimental glomerulonephritis: the modulation of macrophage migration and eicosanoid metabolism. J. Immunol. 143:3192-3199.

9. Lef kowith, J. B., T. Nagamatsu, J. Pippin, and G. F. Schreiner. 1991. Role of leukocytes in metabolic and functional derangements of experimental glomerulonephritis. Am. J. Physiol. 261:F213-F220.

10. Nagamatsu, T., J. Pippin, G. F. Schreiner, and J. B. Lef kowith. 1992. Paradoxical exacerbation of leukocyte-mediated glomerulonephritis with cyclooxygenase inhibition. Am. J. Physiol. 263:F228-F236.

11. Fauler, J., A. Wiemeyer, K. H. Marx, K. Kuhn, K. M. Koch, and J. C. Frolich. 1989. $\mathrm{LTB}_{4}$ in nephrotoxic serum nephritis in rats. Kidney Int. 36:46-50.

12. Stahl, R. A. K., F. Thaiss, S. Kahf, W. Schoeppe, and U. M. Helmchen. 1990. Immune-mediated mesangial cell injury-biosynthesis and function of prostanoids. Kidney Int. 38:273-281.
13. Stork, J. E., and M. J. Dunn. 1983. Hemodynamic role of thromboxane $\mathrm{A}_{2}$ and prostaglandin $\mathrm{E}_{2}$ in glomerulonephritis. J. Pharmacol. Exp. Ther. 233:672-678.

14. Lef kowith, J. B., and G. F. Schreiner. 1987. Essential fatty acid deficiency depletes rat glomeruli of resident mesangial macrophages and inhibits angiotensin II-stimulated eicosanoid synthesis. J. Clin. Invest. 80:947-956.

15. Lef kowith, J. B., A. R. Morrison, and G. F. Schreiner. 1988. Glomerular leukotriene $B_{4}$ synthesis: manipulation by $(n-6)$ fatty acid deprivation and cellular origin. J. Clin. Invest. 82:1655-1660.

16. Lef kowith, J. B., B. A. Jakschik, V. Flippo, and P. Needleman. 1987. Metabolic and functional alterations in macrophages induced by essential fatty acid deficiency. J. Biol. Chem. 262:6668-6675.

17. Cook, H. T., J. Smith, and V. Cattell. 1987. Isolation and characterization of inflammatory leukocytes from glomeruli in an in situ model of glomerulonephritis in the rat. Am. J. Pathol. 126:126-136.

18. Camazine, S. M., G. B. Ryan, E. R. Unanue, and M. J. Karnovsky. 1976. Isolation of phagocytic cells from the rat renal glomerulus. Lab. Invest. 35:315326.

19. Stenson, W. F., and C. W. Parker. 1979. Metabolism of arachidonic acid in ionophore-stimulated neutrophils. J. Clin. Invest. 64:1457-1465.

20. Imai, E., R. L. Hoover, N. Makita, C. D. Funk, and K. F. Badr. 1990. Localization and relative abundance of 5-lipoxygenase, 15-LO, 12-LO, and leukotriene $\mathrm{A}_{4}$ hydrolase gene expression in cultured glomerular cells. J. Am. Soc. Nephrol. 1:443A.

21. Makita, N., C. D. Funk, E. Imai, R. L. Hoover, and K. F. Badr. 1992. Molecular cloning and functional expression of rat leukotriene-A4 hydrolase using the polymerase chain reaction. FEBS (Fed. Eur. Biochem. Soc.) Lett. 299:273-277.

22. Johnson, R. J., C. E. Alpers, P. Pritzl, M. Schulze, P. Baker, C. Pruchno, and W. G. Couser. 1988. Platelets mediate neutrophil-dependent immune complex nephritis in the rat. J. Clin. Invest. 82:1225-1235.

23. Sindrey, M., T. L. Marshall, and P. Naish. 1979. Quantitative assessment of the effects of platelet depletion in the autologous phase of nephrotoxic serum nephritis. Clin. Exp. Immunol. 36:90-96.

24. Cook, H. T., J. Smith, J. A. Salmon, and V. Cattell. 1989. Functional characteristics of macrophages in glomerulonephritis in the rat: $\mathrm{O}_{2}$ generation, MHC class 2 expression and eicosanoid synthesis. Am. J. Pathol. 134:431-437.

25. Osborne, M. J., B. Droz, P. Meyer, and F. Morel. 1975. Angiotensin II: renal localization in glomerular mesangial cells by autoradiography. Kidney Int. 8:245-254.

26. Coyne, D. W., M. Nickols, W. Bertrand, and A. R. Morrison. 1992. Regulation of mesangial cell cyclooxygenase synthesis by cytokines and glucocorticoids. Am. J. Physiol. 263:F97-F102.

27. Remuzzi, G., G. A. Fitzgerald, and C. Patrono. 1992. Thromboxane synthesis and action within the kidney. Kidney Int. 41:1483-1493.

28. Johnson, R. J., P. Pritzl, H. lida, and C. E. Alpers. 1991. Platelet-complement interactions in mesangial proliferative nephritis in the rat. Am. J. Pathol. 138:313-321.

29. Schifferli, J. A., and R. P. Taylor. 1989. Physiological and pathological aspects of circulating immune complexes. Kidney Int. 35:993-1003.

30. Mulligan, M. S., C. G. Yeh, A. R. Rudolph, and P. A. Ward. 1992. Protective effects of soluble CR 1 in complement- and neutrophil-mediated tissue injury. J. Immunol. 148:1479-1485.

31. Ramos, B. F.,Y. Zhang, and B. A. Jakschik. 1992. Mast cells contribute to fibrin deposition in reverse passive Arthus reaction in mouse skin. Eur. J. Immunol. 22:2381-2385.

32. Springer, T. A. 1990. Adhesion receptors of the immune system. Nature (Lond.). 346:425-434. 